\title{
Least squares signal approximation using multirate systems: multichannel nonuniform case
}

\author{
Bojan Vrcelj \\ Dept. of Electrical Engineering \\ California Institute of Technology \\ Pasadena, CA 91125
}

\author{
P. P. Vaidyanathan \\ Dept. of Electrical Engineering \\ California Institute of Technology \\ Pasadena, CA 91125
}

\begin{abstract}
The issue of signal approximation has been treated extensively by the signal processing and mathematics communities. The problem is usually that of minimizing an appropriate metric between the given signal and signals described by a certain model. In this paper we consider the general signal model described by multirate systems. Multiple channels with nonuniform interpolation ratios are considered. We show that the solution minimizing the $\ell_{2}$ norm of the approximation error involves filtering that is in general time-varying. We also point out the conditions under which LTI filters can be used and consider a special uniform case which provides further insights. ${ }^{1}$
\end{abstract}

\section{Introduction}

The problem in signal approximation is invariably that of representing an arbitrary signal by the best approximation from a given, restricted class of signals. The notion of "best" approximation is usually quantitatively described in terms of some metric. An important special case occurs when the signals are squaresummable $\left(\ell_{2}\right.$ signals $)$ and the metric is correspondingly characterized by the $\ell_{2}$ norm. The restricted class of signals in question is sometimes given by a signal model, which is described as the output of a linear system driven by an arbitrary (stable) input.

The flexibility in choosing the signal model leads to applications in several different signal processing areas, such as denoising, sampling theory and multiresolution theory [3]. In spline approximation theory [6], the signal is modeled as the output of a spline interpolation system. This problem was considered in a more general biorthogonal partner setting in [8] and in the case of vector signals the solution is presented in [9]. A closely related subject of oblique projections is ex-

\footnotetext{
${ }^{1}$ Work supported in part by the ONR grant N00014-99-11002, USA.
}

tensively treated in [1]. The signal model considered in this paper is very general since it consists of a bank of interpolation filters with different interpolation ratios. Such structures commonly occur in wavelet theory, and therefore the results presented here play an important role in the theory of approximation in multiresolution spaces. As we shall see, the systems for such approximation in general require the use of timevarying filters.

We begin by describing the problem of signal approximation within nonuniform multichannel signal models. Next, we derive the solution to this problem and describe the conditions for the existence of an LTI solution. Some examples are included to demonstrate that those conditions are often not satisfied. We also consider a special case providing further insights.

\subsection{Notations}

If not stated otherwise, all notations are as in [7]. We use the encircled symbol $\downarrow N$ to denote the decimation operation [turns $x(n)$ into $x(N n)$ ]. The expanded version of $x(n)$

$$
\begin{cases}x(n / N) & \text { for } n=\operatorname{mul} \text { of } N, \\ 0 & \text { otherwise }\end{cases}
$$

is similarly obtained as a result of the expander operation which is denoted by the encircled symbol $\uparrow N$. The decimated and expanded versions of $x(n)$ are denoted by $[x(n)]_{\downarrow N}$ and $[x(n)]_{\uparrow N}$. Superscripts $T$ and $\dagger$ operating on vectors or matrices denote the transpose and the transpose conjugate, respectively. The $\ell_{2}$ norm of $x(n)$ is denoted by $\|x(n)\|$.

\section{Problem formulation and solution}

Consider the model shown in Fig. 1(a). If the filter $F(z)$ is a $B$-spline oversampled by $M$ this model corresponds to spline approximation theory. Given an arbitrary $\ell_{2}$ sequence $x(n)$, a stable filter $F(z)$ and an integer $M>1$ the question is how to find the best approximation $y(n)$ admitting this model? This problem 


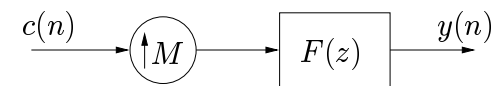

( a )

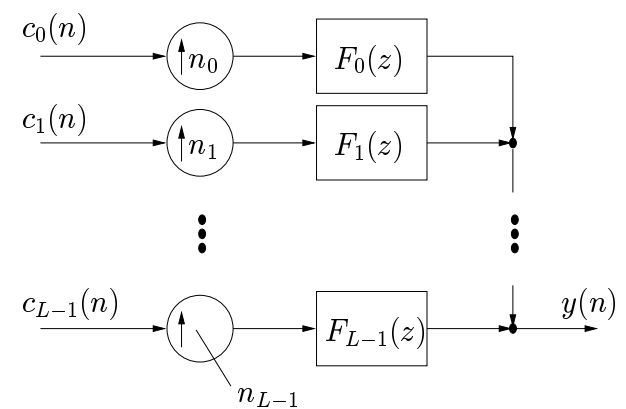

( b )

Figure 1: Signal models: (a) single channel model, (b) multichannel, nonuniform model (see text).

has been solved in the context of biorthogonal partners [8]. Notice that this model also represents the output of a single channel in a nonuniform filter bank.

\subsection{Formulation}

A straightforward extension of the model from Fig. 1 (a) is the $L$-channel model shown in Fig. 1(b). The expanders $\left\{n_{i}\right\}$ across different channels are in general unequal integers. The digital filters $F_{i}(z)$ are given by the impulse responses $f_{i}(n), 0 \leq i \leq L-1$. Any signal $y(n)$ produced by this structure is given by

$$
y(n)=\sum_{i=0}^{L-1} \sum_{k=-\infty}^{\infty} c_{i}(k) f_{i}\left(n-k n_{i}\right) .
$$

By analogy with the previous discussion, the least squares approximation problem is as follows. Given an arbitrary $\ell_{2}$ sequence $x(n)$, the goal is to find the best approximation $y(n)$, admitting the model (1), such that the mean-squared error

$$
\xi \triangleq\|y(n)-x(n)\|^{2}=\sum_{n=-\infty}^{\infty}|y(n)-x(n)|^{2}
$$

is minimized. This problem is evidently equivalent to the problem of finding the optimal sequences $\left\{c_{i}(n)\right\}$, for $0 \leq i \leq L-1$.

\subsection{Solution}

In the following we use several well-known identities from nonuniform filter bank theory treated amply in [5]. Consider the $i$ th channel of the model from Fig. 1(b), redrawn in Fig. 2(a). For any integer $N$ such that $N=k_{i} n_{i}$, where $k_{i}$ is another integer, Fig. 2(a) can be redrawn as in Fig. 2(b) [2]. In particular if
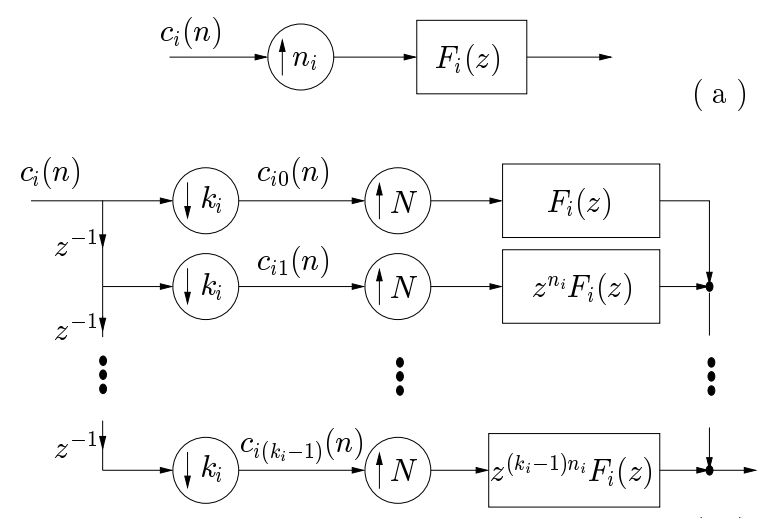

Figure 2: Equivalent single-channel representations.

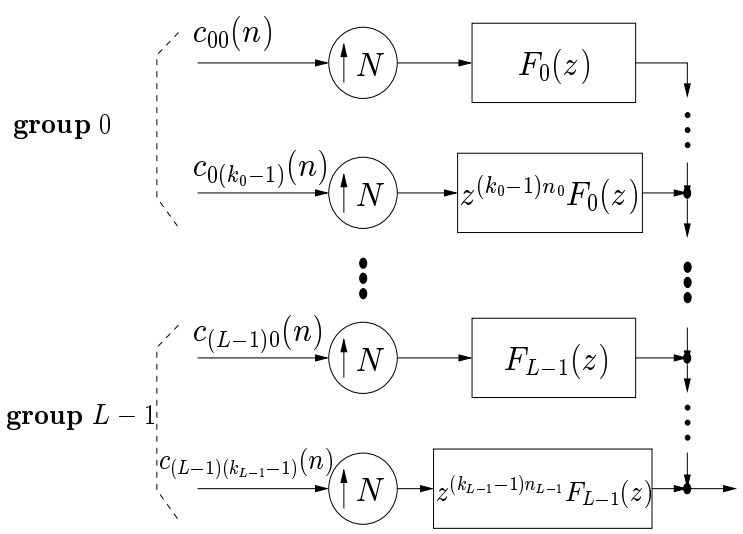

Figure 3: Equivalent uniform signal model.

$$
N=\operatorname{lcm}\left(n_{0}, n_{1}, \ldots, n_{L-1}\right), \text { and } k_{i}=N / n_{i}
$$

this transformation will continue to hold. In the following we assume (3) is satisfied. Repeating the transformation shown in Fig. 2 for all the $L$ channels, we can redraw the nonuniform $L$-channel synthesis bank from Fig. 1(b) as the uniform synthesis bank shown in Fig. 3 consisting of $L$ groups of channels, $k_{i}$ channels in each group. For notational convenience, in the following we often use the integer $K$ denoting the number of channels in Fig. 3; in other words

$$
K \triangleq \sum_{i=0}^{L-1} k_{i}
$$

Notice in Fig. 2(b) that the subsequences $\left\{c_{i j}(n)\right\}$ for $0 \leq j \leq k_{i}-1$ are obtained by parsing the sequence $c_{i}(n)$. Therefore, the problem of finding the optimal driving sequences $\left\{c_{i}(n)\right\}$ in Fig. $1(\mathrm{~b})$ is completely equivalent to that of finding the corresponding 
$K$ inputs $\left\{c_{i j}(n)\right\}$ to the uniform structure from Fig. 3. However, transforming a nonuniform problem to a uniform one, will prove beneficial. Let us denote the filters from Fig. 3 by $P_{i}(z)$, and the corresponding inputs by $b_{i}(n), 0 \leq i \leq K-1$, i.e. let

$$
\begin{aligned}
& P_{0}(z) \triangleq F_{0}(z), \cdots, P_{K-1} \triangleq z^{\left(k_{L-1}-1\right) n_{L-1}} F_{L-1}(z) ; \\
& B_{0}(z) \triangleq C_{00}(z), \cdots, B_{K-1} \triangleq C_{(L-1)\left(k_{L-1}-1\right)}(z) .(5)
\end{aligned}
$$

Now, we can rewrite the error (2) in the frequency domain as follows

$$
\begin{aligned}
\xi & =\int_{0}^{2 \pi}\left|Y\left(e^{j \omega}\right)-X\left(e^{j \omega}\right)\right|^{2} \frac{d \omega}{2 \pi} \\
& =\int_{0}^{2 \pi} \underbrace{\left|\sum_{k=0}^{K-1} P_{k}\left(e^{j \omega}\right) B_{k}\left(e^{j \omega N}\right)-X\left(e^{j \omega}\right)\right|^{2}}_{\mathcal{E}(\omega)} \frac{d \omega}{2 \pi} .
\end{aligned}
$$

Note that $B_{k}\left(e^{j \omega N}\right)$ appearing in the integrand is periodic with period $2 \pi / N$, and therefore can be chosen independently only in the range $0 \leq \omega \leq 2 \pi / N$. Thus the integrand $\mathcal{E}(\omega)$ can be rewritten as

$$
\sum_{n=0}^{N-1}\left|\sum_{k=0}^{K-1} P_{k}\left[e^{j\left(\omega+\frac{2 \pi n}{N}\right)}\right] B_{k}\left(e^{j \omega N}\right)-X\left[e^{j\left(\omega+\frac{2 \pi n}{N}\right)}\right]\right|^{2} .
$$

For each $\omega$ in $0 \leq \omega \leq 2 \pi / N$ we can choose $B_{k}\left(e^{j \omega N}\right)$ such that the nonnegative integrand $\mathcal{E}(\omega)$ is minimized and that would in turn minimize the projection error (2). To solve (6) for the optimal $B_{k}\left(e^{j \omega N}\right), 0 \leq k \leq$ $K-1$, we define the following matrix

$\mathcal{P}(\omega) \triangleq\left[\begin{array}{cccc}P_{0}\left(e^{j \omega}\right) & P_{1}\left(e^{j \omega}\right) & \cdots & P_{K-1}\left(e^{j \omega}\right) \\ P_{0}\left[e^{j\left(\omega+\omega_{1}\right)}\right] & P_{1}\left[e^{j\left(\omega+\omega_{1}\right)}\right] & \cdots & P_{K-1}\left[e^{j\left(\omega+\omega_{1}\right)}\right] \\ \vdots & \vdots & \vdots & \vdots \\ P_{0}\left[e^{j\left(\omega+\omega_{N-1}\right)}\right] & P_{1}\left[e^{j\left(\omega+\omega_{N-1}\right)}\right] & \cdots & P_{K-1}\left[e^{j\left(\omega+\omega_{N-1}\right)}\right]\end{array}\right]$

for $\omega_{n}=\frac{2 \pi n}{N}$. Also we define the following vectors

$$
\begin{aligned}
& \mathcal{B}(N \omega) \triangleq\left[\begin{array}{llll}
B_{0}\left(e^{j \omega N}\right) & B_{1}\left(e^{j \omega N}\right) & \cdots & B_{K-1}\left(e^{j \omega N}\right)
\end{array}\right]^{T}, \\
& \mathcal{X}(\omega) \triangleq\left[\begin{array}{lll}
X\left(e^{j \omega}\right) & X\left[e^{j\left(\omega+\frac{2 \pi}{N}\right)}\right] & X\left[e^{j\left(\omega+\frac{2 \pi(N-1)}{N}\right)}\right.
\end{array}\right]^{T} \cdot(8)
\end{aligned}
$$

Now, we can rewrite (6) as

$$
\mathcal{E}(\omega)=\|\mathcal{P}(\omega) \cdot \mathcal{B}(N \omega)-\mathcal{X}(\omega)\|^{2} .
$$

Our task is to minimize (9) pointwise in $\omega$ by choosing the optimal $\mathcal{B}(N \omega)$. We achieve this by rewriting (9) as follows

$$
\begin{aligned}
\mathcal{E}(\omega)= & {\left[\mathcal{B}^{\dagger}(N \omega)-\mathcal{X}^{\dagger}(\omega) \mathcal{P}(\omega) \mathbf{S}^{-1}(\omega)\right] \mathbf{S}(\omega) \cdot } \\
& \cdot\left[\mathcal{B}(N \omega)-\mathbf{S}^{-1}(\omega) \mathcal{P}^{\dagger}(\omega) \mathcal{X}(\omega)\right]+\mathcal{X}^{\dagger}(\omega) \mathcal{X}(\omega) \\
& -\mathcal{X}^{\dagger}(\omega) \mathcal{P}(\omega) \mathbf{S}^{-1}(\omega) \mathcal{P}^{\dagger}(\omega) \mathcal{X}(\omega),
\end{aligned}
$$

where $\mathbf{S}(\omega) \triangleq \mathcal{P}^{\dagger}(\omega) \mathcal{P}(\omega)$. Consider the right hand side of the equality in (10). It consists of two parts; the first part depends on the choice of $\mathcal{B}(N \omega)$ and the second part does not. Since the first part is always nonnegative, we should choose $\mathcal{B}(N \omega)$ such that it becomes zero. In the following we assume that

$$
\left[\left|P_{k}\left(e^{j \omega}\right)\right|^{2}\right]_{\downarrow N}>0, \text { for } 0 \leq k \leq K-1 .
$$

It follows that the matrix $\mathbf{S}(\omega)=\mathcal{P}^{\dagger}(\omega) \mathcal{P}(\omega)$ is positive definite and therefore, the only way to make the first part of the right hand side in (10) zero is to choose

$$
\mathcal{B}(N \omega)=\left[\mathcal{P}^{\dagger}(\omega) \mathcal{P}(\omega)\right]^{-1} \mathcal{P}^{\dagger}(\omega) \cdot \mathcal{X}(\omega) .
$$

In order to rewrite this solution in terms of the multirate building blocks, let us determine the $(i, j)$ th element of the matrix $\mathcal{P}^{\dagger}(\omega) \mathcal{P}(\omega)$ and the $j$ th element of the vector $\mathcal{P}^{\dagger}(\omega) \cdot \mathcal{X}(\omega)$

$$
\begin{aligned}
{\left[\mathcal{P}^{\dagger}(\omega) \mathcal{P}(\omega)\right]_{i, j} } & =\sum_{n=0}^{N-1} P_{i}^{*}\left[e^{j\left(\omega+\frac{2 \pi n}{N}\right)}\right] P_{j}\left[e^{j\left(\omega+\frac{2 \pi n}{N}\right)}\right] \\
& =N \cdot\left[P_{i}^{*}\left(e^{j \omega}\right) P_{j}\left(e^{j \omega}\right)\right]_{\downarrow N \uparrow N}, \\
{\left[\mathcal{P}^{\dagger}(\omega) \mathcal{X}(\omega)\right]_{j} } & =\sum_{n=0}^{N-1} P_{j}^{*}\left[e^{j\left(\omega+\frac{2 \pi n}{N}\right)}\right] \mathcal{X}\left[e^{j\left(\omega+\frac{2 \pi n}{N}\right)}\right] \\
& =N \cdot\left[P_{j}^{*}\left(e^{j \omega}\right) \mathcal{X}\left(e^{j \omega}\right)\right]_{\downarrow N \uparrow N} .
\end{aligned}
$$

Next, define the row vector

$$
\mathbf{p}\left(e^{j \omega}\right) \triangleq\left[P_{0}\left(e^{j \omega}\right) \quad P_{1}\left(e^{j \omega}\right) \quad \cdots \quad P_{K-1}\left(e^{j \omega}\right)\right] .
$$

Then from (12), keeping (14) in mind, we have that the solution for the optimal subsequences $\left\{b_{k}(n)\right\}$ is given by

$$
\mathcal{B}(\omega)=\left[\left(\left[\mathbf{p}^{\dagger}\left(e^{j \omega}\right) \mathbf{p}\left(e^{j \omega}\right)\right]_{\downarrow N \uparrow N}\right)^{-1} \mathbf{p}^{\dagger}\left(e^{j \omega}\right) \cdot X\left(e^{j \omega}\right)\right]_{\downarrow N}
$$

Recalling the definition of the sequences $\left\{b_{k}(n)\right\}$ from (5), we can represent the solution (15) as shown in Fig. 4(a). The $K \times 1$ vector $\mathbf{h}(z)$ containing the transfer functions of the projection prefilters $H_{i j}(z), 0 \leq i \leq$ $L-1$ and $0 \leq j \leq k_{i}-1$ can be determined from (15) $\operatorname{as}^{2}$

$$
\mathbf{h}(z)=\left([\tilde{\mathbf{p}}(z) \cdot \mathbf{p}(z)]_{\downarrow N \uparrow N}\right)^{-1} \tilde{\mathbf{p}}(z) .
$$

As another remark, notice that the matrix $\left[\mathbf{p}^{\dagger}\left(e^{j \omega}\right) \cdot \mathbf{p}\left(e^{j \omega}\right)\right]_{\downarrow N \uparrow N}$ appearing in the solution is invertible as long as the condition (11) is satisfied.

\footnotetext{
${ }^{2} \tilde{\mathbf{p}}(z)=\mathbf{p}^{* T}\left(1 / z^{*}\right)$.
} 


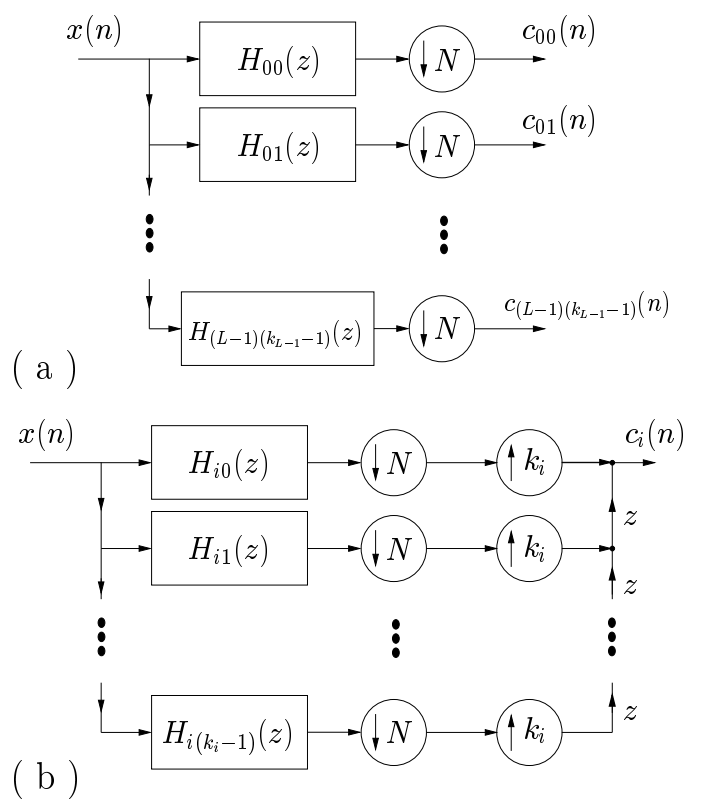

Figure 4: (a) The solution to the approximation problem in terms of the equivalent uniform system. (b) Recovering $\left\{c_{i}(n)\right\}$ from subsequences.

Having obtained the optimal subsequences as in Fig. 4(a), we can recover the optimal driving sequences $\left\{c_{i}(n)\right\}$ in Fig. 1(b) by blocking $\left\{c_{i j}(n)\right\}$. This is shown in Fig. 4(b). Using simple multirate manipulations, we can redraw this structure as in Fig. 5 (a) [recall that $k_{i} n_{i}=N$ ]. Notice that the system shown in a dashed box in Fig. 5(a) is linear, periodically time-varying with period $N$ [denoted by $\operatorname{LPTV}(N)]$ and $k_{i}$ components $H_{i j}(z), 0 \leq j \leq k_{i}-1$. This time-varying filter is denoted by $H_{i}(z, n)$ in Fig. 5 (b) [index $n$ denotes the dependence on time]. Incorporating the simplified representation from Fig. 5(b) into the solution structure in Fig. 4, we see that the system for projection prefiltering (finding the optimal driving sequences $\left.\left\{c_{i}(n)\right\}\right)$ is as shown in Fig. 6 . Once determined, $\left\{c_{i}(n)\right\}$ should be fed into the signal model in Fig. 1(b) to produce the optimal approximation $y(n)$ closest (in $\ell_{2}$ norm) to the given $x(n)$.

From Fig. 5(a) one can conclude that in order for $H_{i}(z, n)$ to be time-invariant, the components $H_{i j}(z)$ that are "active" at different time instances need to be the same (with appropriate time shifts). More specifically, the necessary and sufficient conditions for $H_{i}(z, n)$ to be time-invariant (LTI) are given by

$$
H_{i m}(z)=z^{-n_{i} m} \cdot H_{i 0}(z) \text {, for } 0 \leq m \leq k_{i}-1 \text {. }
$$

Even though the vector $\mathbf{p}(z)$ appearing in the solution (16) has a special structure in that many of its com-

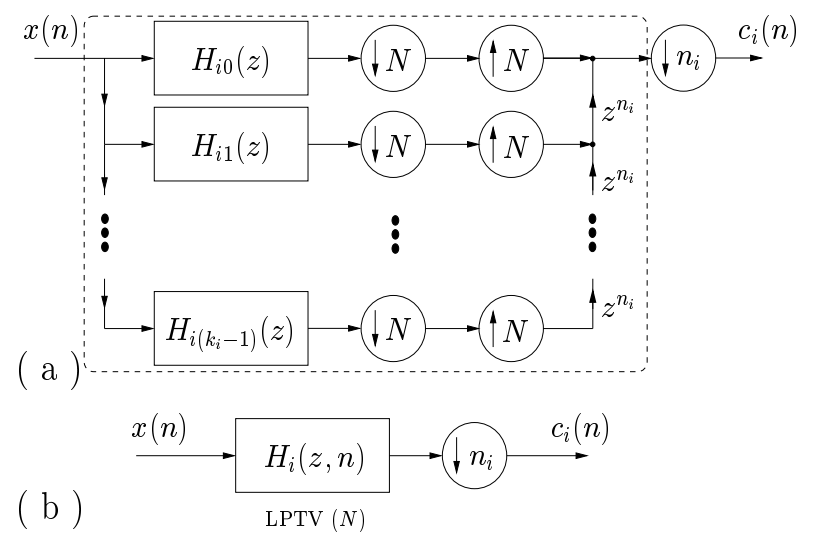

Figure 5: (a) Equivalent drawing of Fig. 4(b). (b) Notation for the time-varying system.

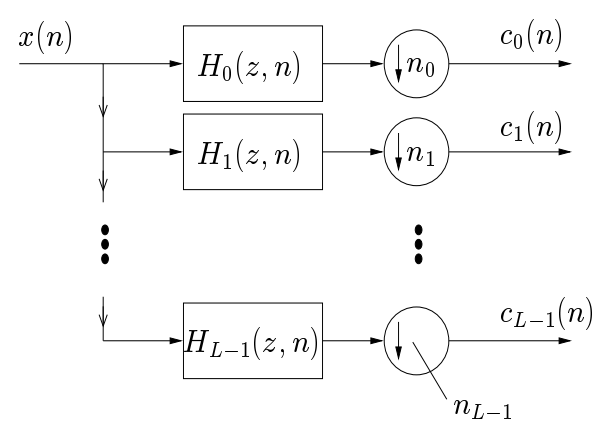

Figure 6: Solution to the approximation problem.

ponents are time-shifts of each other, this is still not sufficient for the LTI conditions (17) to be satisfied in general. This claim will be demonstrated by examples in the next section.

One situation where the LTI conditions are guaranteed to be satisfied is when all the expanders $n_{i}$ are equal: $n_{0}=n_{1}=\cdots=n_{L-1}=N$. This is a special uniform case. The signal model is then similar to that shown in Fig. 3, only with $L$ channels and filters $\left\{F_{i}(z)\right\}, 0 \leq i \leq L-1$. Going through the same steps (5)-(15) it is not hard to see that the solution in this case is again given by (16), only the elements of $\mathbf{h}(z)$ are now projection prefilters $H_{i}(z)$ and not components of their time-varying versions as in the previous discussion. Thus the solution is always LTI.

\section{Examples of LPTV solutions}

Consider the three channel filter bank shown in Fig. 7 , where the filters $F_{i}(z)$ and the decimators $n_{i}$ are fixed. If all three channels are present and the decimators are $\{2,4,4\}$ we know [4] that there exists an LTI analysis bank $\left\{H_{i}(z)\right\}$ such that the whole system has 


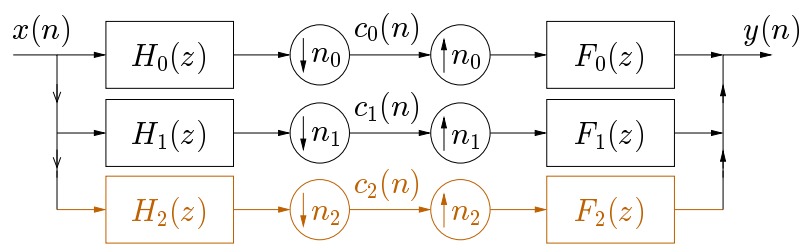

Figure 7: Three channel nonuniform filter bank used in examples. See text.
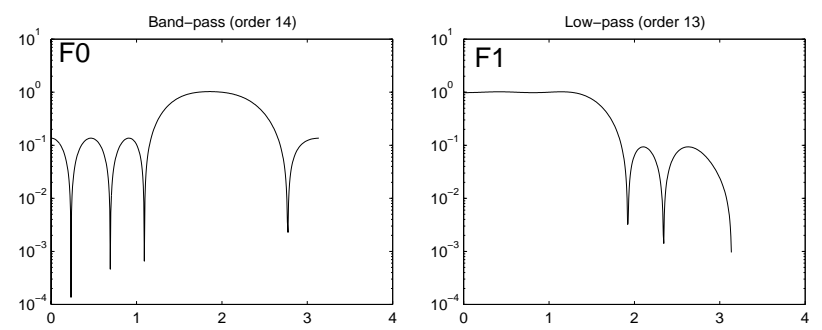

Figure 8: The model filters $F_{0}(z)$ and $F_{1}(z)$.

perfect reconstruction (PR) property. Now, suppose we keep only the first two channels (shown in darker ink), with decimators $n_{0}=2, n_{1}=4$ and filters $F_{0}(z)$, $F_{1}(z)$ shown in Fig. 8. These two synthesis channels define a nonuniform signal model [see Fig. 1(b)]. The solution for the optimal projection prefilters $H_{i j}(z)$ is as in Fig. 4(a). In this case there are only three filters $H_{00}(z), H_{01}(z)$ and $H_{10}(z)=H_{1}(z)$ in the equivalent uniform system. The impulse responses $h_{00}(n)$ and $h_{01}(n)$, for $n \leq 65$ are shown in Fig. 9. A closer look at Fig. 9 reveals that in this example $H_{00}(z)$ and $H_{01}(z)$ do not satisfy (17) and thus the corresponding filter $H_{0}(z, n)$ is necessarily LPTV.

As a more general example, consider again Fig. 7 with decimators $\{2,3,6\}$. Since these decimators violate the compatibility condition [4], for any choice of rational $\left\{F_{i}(z)\right\}$ there are no corresponding rational LTI filters $\left\{H_{i}(z)\right\}$ that make the complete system time-invariant. In particular if $F_{3}(z)=0$ the previous assertion implies that when $n_{0}=2$ and $n_{1}=3$ the LTI conditions for the optimal projection filters (in a two channel model with rational filters) can never be satisfied [regardless of $F_{0}(z)$ and $F_{1}(z)$ ].

\section{Concluding remarks}

In this paper we consider the problem of leastsquares signal approximation using the multichannel models with nonuniform expanders. We show that the solution for the optimal prefilters in general uses timevarying systems. This is demonstrated by two examples. However, in the special case when the expanders
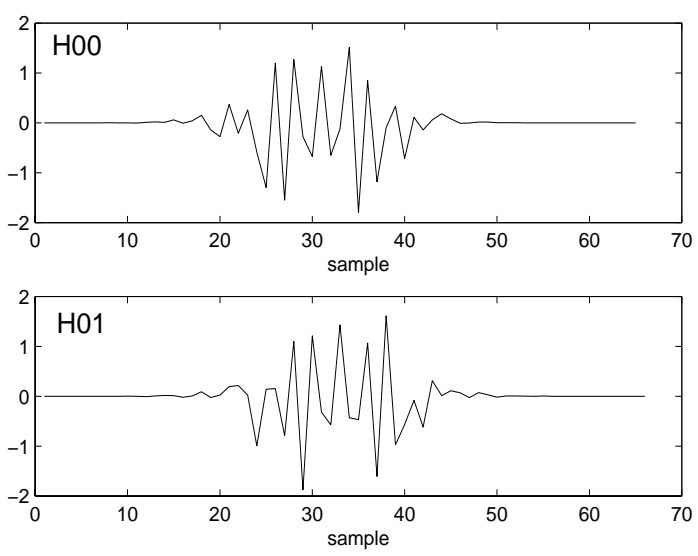

Figure 9: Projection prefilters $H_{00}(z)$ and $H_{01}(z)$ corresponding to the model filters $F_{0}(z)$ and $F_{1}(z)$.

across different channels are equal, the optimal prefilters are indeed LTI.

\section{References}

[1] A. Aldroubi and M. Unser, "Oblique projections in discrete signal subspaces of $\ell_{2}$ and the wavelet transform," Proc. SPIE, vol. 2303, Wav. appl. in signal and image proc., II, San Diego, CA, 1994.

[2] P.-Q. Hoang and P. P. Vaidyanathan, "Nonuniform multirate filter banks: theory and design," Proc. ISCAS, Portland, OR, May 1989.

[3] S. Mallat, A Wavelet Tour of Signal Processing, Academic Press, London, 1998.

[4] S. Akkarakaran and P. P. Vaidyanathan, "New results and open problems on nonuniform filterbanks," Proc. ICASSP, Phoenix, AZ, Mar. 1999.

[5] S. Akkarakaran and P. P. Vaidyanathan, "Nonuniform filter banks: new results and open problems," in Beyond Wavelets, J. Stoecker and G. V. Welland, Eds., Academic Press (in prep.)

[6] M. Unser, A. Aldroubi and M. Eden, "B-spline signal processing: Part I - Theory," IEEE Trans. Signal Processing, vol. 41, pp. 821-833, Feb. 1993.

[7] P. P. Vaidyanathan, Multirate Systems and Filter Banks. Prentice-Hall, Englewood Cliffs, NJ, 1995.

[8] P. P. Vaidyanathan and B. Vrcelj, "Biorthogonal partners and applications," IEEE Trans. Signal Processing, vol. 49(5), pp. 1013-1027, May 2001.

[9] B. Vrcelj and P. P. Vaidyanathan, "Results on vector biorthogonal partners," Proceedings ICASSP, Salt Lake City, Utah, May 2001. 